\title{
Effect of the Cooling Rate on the Graphite Nodule Count and Size Distribution in Nodular Cast Iron
}

\author{
Haji Muhammad Muhmond ${ }^{\mathrm{a},{ }^{*}}$, Hasse Fredriksson ${ }^{\mathrm{a}, \mathrm{b}}$ \\ ${ }^{a}$ Frecast $A B$, Hägersten, Sweden \\ ${ }^{\mathrm{b}}$ Royal Institute of Technology (KTH), Stockholm, Sweden \\ ahaji@kth.se, bhassef@kth.se
}

\begin{abstract}
Keywords: Nodule size distribution, nodule count, cooling rate, nodular cast iron, MgO nucleation, micro segregation.
\end{abstract}

\begin{abstract}
The graphite nodule count, size distribution and homogenization of the nodules distribution are the factors which are of more significance for the properties of the material. By just increasing the inoculants or $\mathrm{Mg}$ will not help to get rid of problems like the un-even size distribution and or increasing the nodule count. The cooling conditions of the melt prior to solidification and during solidification can control these two parameters to a large extent. In this research, it is more emphasized on the nucleation sequence of $\mathrm{MgO}$ particles and on the cooling rates. The nucleation of $\mathrm{MgO}$ at different temperatures and at different cooling rates was found to have a great influence on the nodule size distribution and the homogenization of the microstructure. A mathematical model was derived to relate the $\mathrm{Mg}$ concentration in the liquid to the cooling rate, prior to solidification. The $\mathrm{MgO}$ particles count was calculated as a function of cooling rate. It was found that at higher cooling rates, $\mathrm{MgO}$ can be nucleated in multi steps during cooling process, which can increase the nodule count tremendously.
\end{abstract}

\section{Introduction}

Cast iron is a composite type of alloy in which the graphite shape, size, count and distribution, all are affecting the mechanical and thermal properties of the alloy. At a longer solidification intervals, such as in wind turbine, the micro segregation plays a dominant role on the graphite morphology, thus resulting in non-homogeneity in the microstructure from surface towards the centre of the casting. If the degree of non-homogeneity is higher, then the mechanical properties can be affected but if it exist locally in small areas then it might not have much influence on the overall mechanical behaviour. Improper nucleation sequence of graphite nodule and micro segregation of different elements, can cause the non-homogeneous distribution of nodules and colonies of various sizes are formed. If the average nearest neighbour graphite nodule spacing is lower among the nodules in a colony than the nodules that are formed at early stage of solidification. According to Kobayashi et. al [1], the increased distance among graphite nodules leads to increased plastic strain for coalescence of voids, therefore fracture toughness increases. In the areas where many nodules are formed at the same time as a colony, as in the last solidifying regions, the inter-nodular spacing will be lower and the fracture toughness will decrease. The larger is the size of the colony with many nodules, the lower will be the fracture toughness, thus behaving like a local defect in the material.

The nucleation of graphite nodules has been investigated by many researchers [2, 3 and 4$]$ and there are many evidences that $\mathrm{MgO}$ is the most effective and commonly found nucleating substrate for the graphite nodule. There are various other oxides/sulphides such as $\mathrm{CaO} / \mathrm{S}$, Rare Earth (RE)oxides etc., but from mass balance one can find that about $95 \%$ will be $\mathrm{MgO}$ and the remaining $5 \%$ will contain $\mathrm{Ca}$ and $\mathrm{RE}$-oxides. The role of $\mathrm{MgO}$ particle is dominant regardless of the fact that the melt is treated with inoculants or not. Therefore one can conclude that $\mathrm{MgO}$ is playing $95 \%$ role in providing nucleation sites for graphite nodules, either homogeneously or heterogeneously. In fact the remaining $5 \%$ oxides are also bonded with $\mathrm{MgO}$ to form complex oxides, since $\mathrm{Ca}$ and $\mathrm{RE}$ - oxides are formed at the very early stage of adding them to the melt. Therefore $\mathrm{MgO}$ will 
nucleate heterogeneously on them. When all of the $\mathrm{Ca}$ and $\mathrm{RE}$ elements are utilized, then $\mathrm{MgO}$ will nucleate either homogeneously or heterogeneously. Therefore the nucleation sequence of $\mathrm{MgO}$ and amount of $\mathrm{MgO}$ particles will affect greatly the count, size and distribution of graphite nodules.

SKB, Swedish Nuclear Fuel and Waste Management Company, is tasked with managing Swedish nuclear and radioactive waste in a safe way. SKB uses a special method for final disposal of the spent nuclear fuel, called KBS-3. One important component in this method is the load bearing nodular cast iron insert. The insert is nearly $5 \mathrm{~m}$ high and $1 \mathrm{~m}$ in diameter. Two different design are used. The PWR-insert are designed with 4 channels to host fuels from Pressure Water Reactors and the BWR-inserts have 12 channels for fuel from Boiling Water Reactors. In this report, samples from two different test manufactured inserts have been investigated for the variation in the graphite structure.

A detail study was performed on these samples such as porosity distribution and comparison in the mechanical properties between different parts, but only the information regarding the graphite structure in some selected samples, is included here. Finally a mathematical model is derived with the help of thermodynamics of the formation of Mg-oxides and diffusion driven growth of particles. This model will describe the formation of $\mathrm{MgO}$ particles at different cooling rates. The current model is valid from the inoculation temperature till the starting point of solidification. This model could tell us about the number of $\mathrm{MgO}$ particles that are formed at different cooling rates. In near future, model will be able to evaluate the $\mathrm{MgO}$ nucleation and graphite nodule growth at variable cooling rates during solidification.

\section{Material and Experiments}

Samples from one PWR-insert (IP24) and one BWR-insert (I76) have been investigated. The composition of the two inserts is provided in Table 1. Inserts cross-section is shown below schematically in Figure 1. These two insert have different cooling conditions. Samples were taken $1150 \mathrm{~mm}$ from the bottom end and at position number 3, 4 and 7 from PWR-insert and position 2 and 7 from the middle of BWR-insert. The samples were cut from the tensile test bars. All samples were polished down to $1 \mu \mathrm{m}$ size diamond suspension. Few samples were chemically etched with Nital $4 \%$ solution. Colour etching was performed with hot alkaline solution (28 $\mathrm{g} \mathrm{NaOH}, 1 \mathrm{~g} \mathrm{KOH}, 200 \mathrm{ml} \mathrm{H}_{2} \mathrm{O}$ and $4 \mathrm{~g}$ Picric acid).

Table 1. Composition of the alloys.

\begin{tabular}{l|cccccccccccc} 
Alloys & $\mathrm{C}$ & $\mathrm{Si}$ & $\mathrm{Mn}$ & $\mathrm{P}$ & $\mathrm{S}$ & $\mathrm{Cr}$ & $\mathrm{Ni}$ & $\mathrm{Cu}$ & $\mathrm{Mg}$ & $\mathrm{Sb}$ & $\mathrm{Fe}$ & $\mathrm{CE}$ \\
\hline IP24 & 3.59 & 2.2 & 0.12 & 0.01 & 0.006 & 0.03 & 0.38 & 0.03 & 0.046 & 0.004 & 93.5 & 4.32 \\
I76 & 3.50 & 2.18 & 0.17 & 0.02 & 0.006 & 0.03 & 0.39 & 0.02 & 0.045 & 0.003 & 93.64 & 4.23
\end{tabular}

\section{Results}

The material used was treated with FeSiMg and pre-inoculated with commercially available inoculants. The microstructure study of samples from insert BWR and PWR showed a large variation in the graphite nodule size and distribution close to the steel channels and away from the steel channels or in other words, fast cooling areas and slow cooling areas. The overall solidification time was calculated in a separate report by A. Tadesse [5] by using Comsole Multiphysics for both PWR and BWR inserts and it was shown that the it took about 11 hours and 2 hours respectively for the insert to be completely solidified. The last solidified region in PWR occurred at sample position 7, whereas in BWR it lied at sample position number 2. 


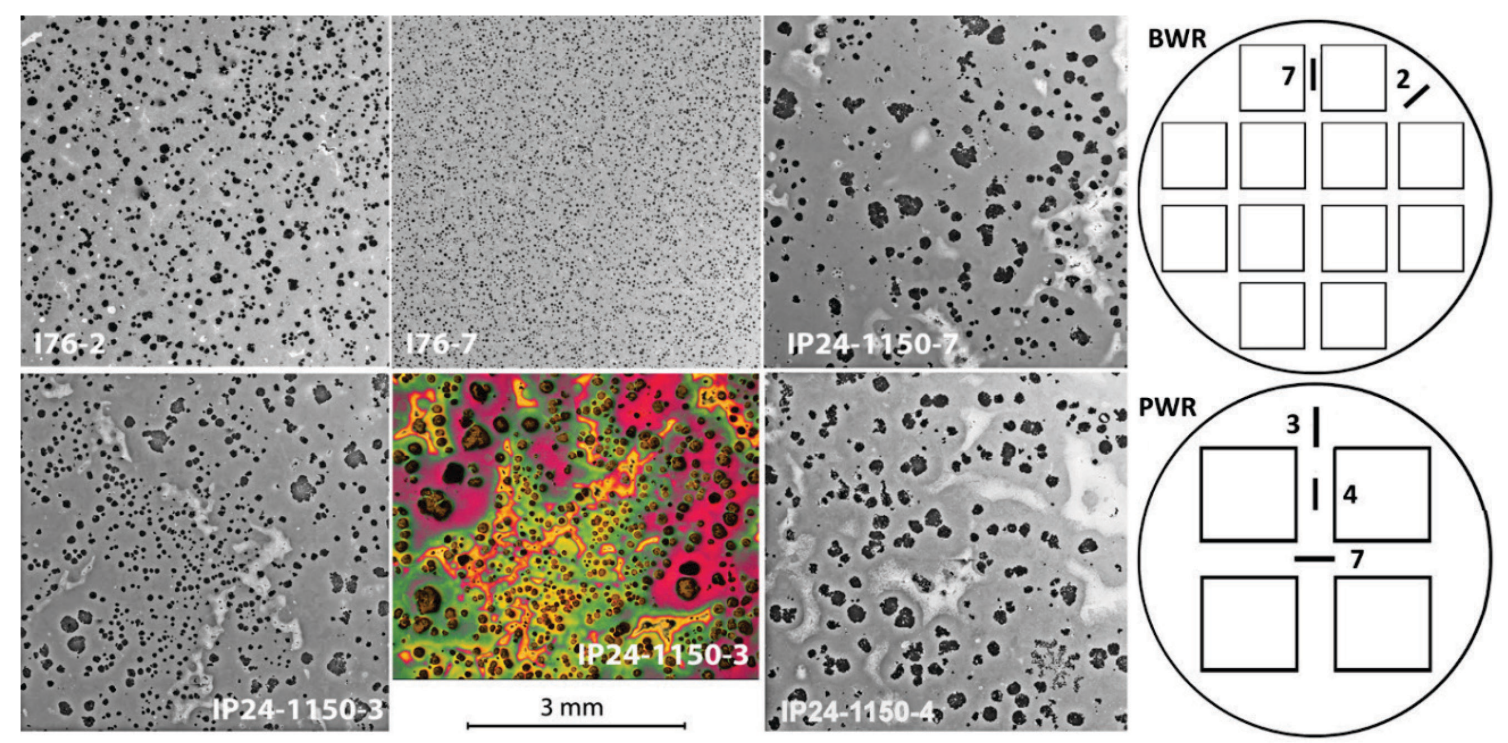

Fig. 1. Microstructure of graphite in the samples from PWR and BWR inserts and schematic Cross section view of the inserts and samples positions. All images are at the same magnification.
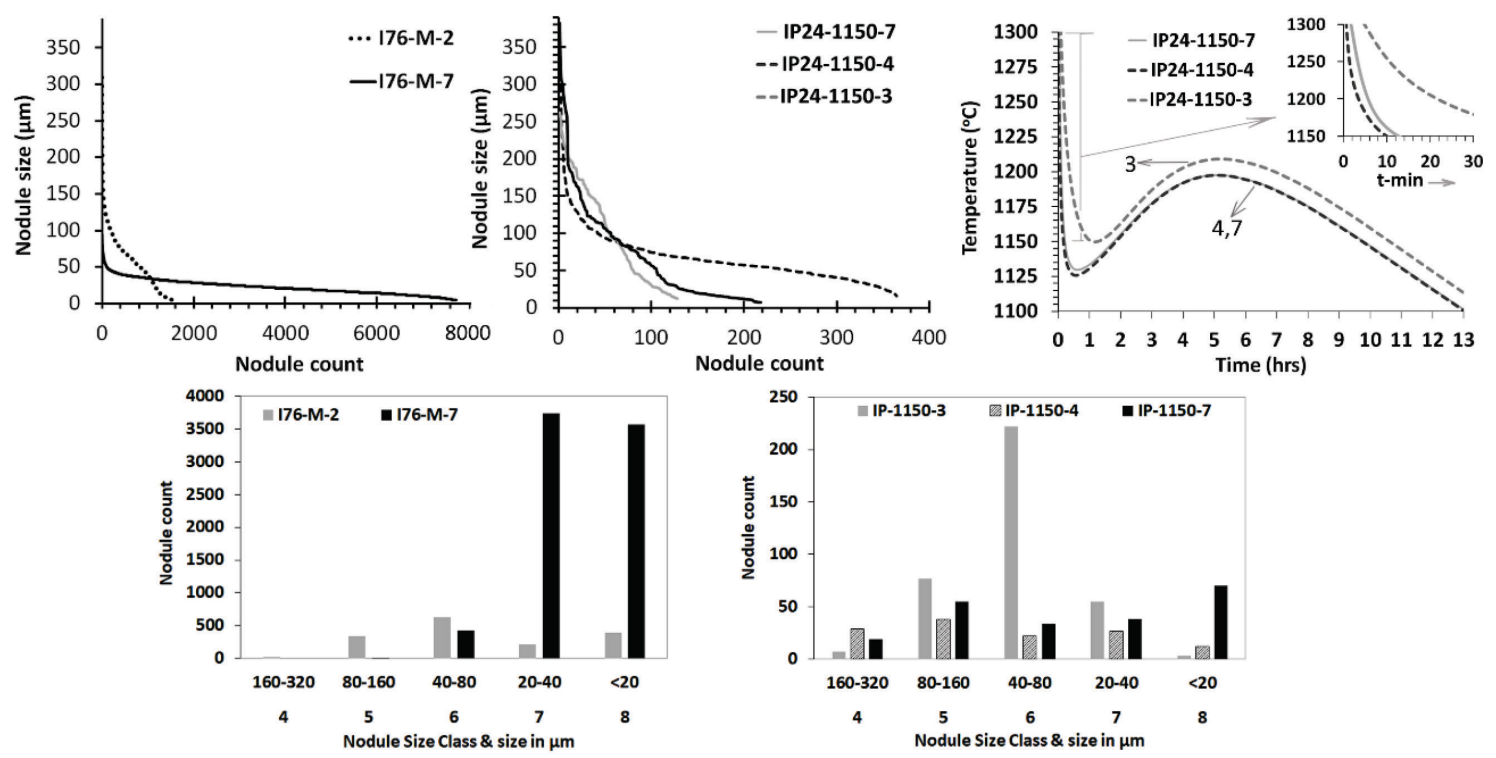

Fig. 2. Nodule count and size distribution in the samples from PWR and BWR inserts. Nodule size class is according to ASTM A247-16a standard. Simulated cooling curve for 1150-3, 4 and 7 [5].

Statistical analyses were performed on the samples at position shown in Figure 1 for both the inserts. In BWR, samples at position 7 has much higher cooling rate compared to sample in position 2. The variation in the nodule size distribution can be observed in Figure 2. From this comparison one can find that the nodule count increased enormously at a higher cooling rate. For example sample I76-7 have 5 times higher nodule count than in the sample I76-2. 95\% of the nodules are less than $40 \mu \mathrm{m}$ in size among which almost half are less than $20 \mu \mathrm{m}$ in size.

In PWR insert, the nodule count varies less between different positions, compared to BWR due to overall longer solidification time (Figure 2). However there is a much larger variation in the nodule size distribution within the samples as in number 3 and 7. There are large segregation areas in these samples as shown by the colour etched figure for sample 3. There are different size colonies of graphite nodules. The small size nodule are formed at the end of solidification. Sample at position 7 in PWR is at the longest cooling time condition having much larger segregation areas. The number of graphite nodule are less than in sample 3. Graphite nodule size in sample 4 have grown to almost the same size as in sample 3 or 7 with a single size graphite colony. There are some small size nodules but they are uniformly distributed. 


\section{Discussion}

Mathematical calculations are performed to predict the nucleation of $\mathrm{MgO}$ at different but constant cooling rates; however, there is never constant heat transfer in the mould. The cooling rate always decreases immediately after the casting operation. The diffusion controlled growth rate of $\mathrm{MgO}$ is calculate by using equation 1 .

$$
d r / d t=D_{M g} * V_{m}^{M g O} / V_{m}^{M g} r\left(\left(X_{M g}^{e q}-X_{M g}^{L}\right) /\left(X_{M g}^{e q}-X_{M g}^{M g o}\right)\right)
$$

Where $X_{M g}^{e q}$ is the equilibrium amount of $\mathrm{Mg}$ in the liquid with $\mathrm{MgO}, X_{M g}^{L}$ is the $\mathrm{Mg}$ content in the melt which varies during cooling, $X_{M g}^{M g O}$ is the $\mathrm{Mg}$ content in the $\mathrm{MgO}$ particle which is assumed to 0.5 mole fraction. $V_{m}^{M g O}$ is the molar volume of $\mathrm{MgO}$ particle $\left(1.14 \times 10^{-5} \mathrm{~m}^{3} / \mathrm{mol}\right), V_{m}^{M g}$ is the molar volume of $\mathrm{Mg}$ in the liquid in equilibrium $\left(1.09143 \times 10^{-9} \mathrm{~T}+6.08 \mathrm{~m}^{3} / \mathrm{mol}\right.$ [6], $D_{M g}$ is the diffusion constant for $\mathrm{Mg}$ in the melt which is assumed as $1 \times 10^{-11} \mathrm{~m}^{2} / \mathrm{s}$ and ' $\mathrm{r}$ ' is the radius of $\mathrm{MgO}$ particle at the time of inoculation. The equilibrium $\mathrm{Mg}$ content ' $X_{M g}^{L}$ ' varies according to the growth of $\mathrm{MgO}$ particles and the initial concentration of $\mathrm{Mg}\left(X_{M g}^{o}\right)$. It is calculated by using equation 2 .

$$
\begin{aligned}
X_{M g}^{e q}= & 1 /\left(X_{O}^{e q} f_{O} f_{M g}\right) * \exp (-\Delta G / R T) \\
& X_{M g(t)}^{L}=X_{M g}^{o}-V_{m}^{M g} / V_{m}^{M g o}\left(\left(N\left(\frac{2}{3}\right) \pi R^{3}\right) /\left(1-N \frac{4}{3} \pi R^{3}\right)\right)
\end{aligned}
$$

' $\mathrm{N}$ ' is the number of $\mathrm{MgO}$ particles that are formed in the melt. $X_{M g}^{o}$ is the initial $\mathrm{Mg}$ concentration in the liquid. When $X_{M g(t)}^{L}$ reaches the supersaturation limit where $\mathrm{MgO}$ can be nucleated, the number of $\mathrm{MgO}$ particles will get doubled. This number can get 2, 4, 8, 16, and 32 times depending how many times the super saturation limit is achieved. At each nucleation step, the $X_{M g}^{o}$ will be adjusted in such a way that the $\mathrm{Mg}$ level in the liquid will reach back to the equilibrium $\mathrm{Mg}$ concentration. The homogeneous nucleation of $\mathrm{MgO}$ can be evaluated by using equation 4. Surface tension was assumed 0.7 to $0.3 \mathrm{~J} / \mathrm{m}^{2}$ from 1450 down to $1150{ }^{\circ} \mathrm{C}$.

$$
X_{M g}^{n u c}=X_{M g}^{e q} \exp \left(\sqrt{\left(16 \pi \sigma^{3} V_{m}^{2}\right) /\left(180 K_{B} T\right)}\right) / R T
$$

The results shown in Figure 3 indicate that by increasing the cooling rates of the melt (prior to solidification in this article), The Mg level suffers a little change and the slope of this change is greater at higher cooling rates. The faster is the cooling rate, the quickest it will reach the nucleation line where new $\mathrm{MgO}$ particles can be nucleated homogeneously. However; at slow cooling, the $\mathrm{Mg}$ level go side by side the equilibrium line and it may not reach the nucleation line prior to solidification, shown in Figure 3 (a). The Mg level could reach the nucleation line either once or twice, or even multiple times, depending on the cooling rate. At each nucleation event, the number of $\mathrm{MgO}$ particles will get doubled as shown in Figure 3(f). Due to this, the growth rate of the particle will reduce and as shown in Figure 3(e).

In our experiments it was observed that the cooling rate is not constant. The above calculations are valid for a constant cooling rate. For a variable cooling rate, as shown by an example in Figure 3 (d) where the cooling rate was reduced abruptly from a constant rate of $100 \mathrm{~K} / \mathrm{min}$ to $20 \mathrm{~K} / \mathrm{min}$, the $\mathrm{Mg}$ level again starts to follow the equilibrium line and no more $\mathrm{MgO}$ can be nucleated prior to solidification starts. Also the existing nuclei which are small in size, like those which are nucleated in the $2^{\text {nd }}$ and $3^{\text {rd }}$ nucleation event, might get unstable and dissolve back in the melt due to change in composition. During the solidification, due to micro segregation of all elements, there will be again possibility to nucleate new particles as shown by Muhmond et. Al [4]. Based on the above results, the solidification mechanism or the nucleation mechanism can be divided into two cases. 

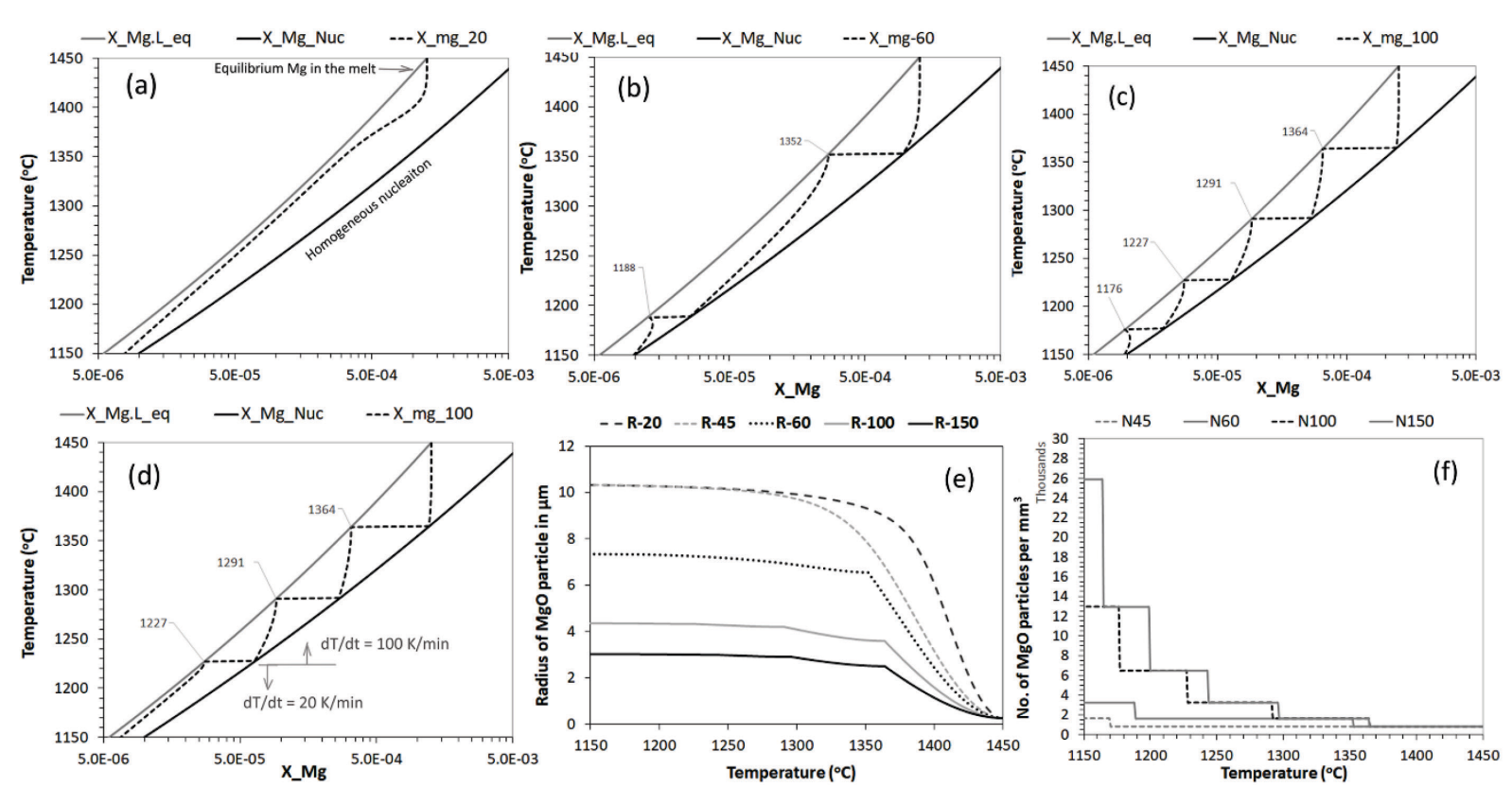

Figure 3. (a-d) The change in the $\mathrm{Mg}$ concentration during at different cooling rates whereas (a) $20 \mathrm{~K} / \mathrm{min}$ (b) $60 \mathrm{~K} / \mathrm{min}$ (c) $100 \mathrm{~K} / \mathrm{min}$ (d) $100 \mathrm{~K} / \mathrm{min}$ down to $1227^{\circ} \mathrm{C}$ but after that $20 \mathrm{~K} / \mathrm{min}$ till $1150{ }^{\circ} \mathrm{C}$, (e) The change in the size of $\mathrm{MgO}$ particle which is nucleation homogeneously, at different cooing rates $\mathrm{R}-20$ to $\mathrm{R}-150 \mathrm{~K} / \mathrm{min}$. (f) is the number of $\mathrm{MgO}$ particles as a function of cooling rates.

Case I: Constant and gradual decrease in cooling rate: In this case the cooling rate is either constant or decreasing gradually with small steps, throughout the cooling and during solidification. In the slow cooling process, the nucleation of $\mathrm{MgO}$ would occur either once or never in the liquid prior to solidification; however during solidification, there will be a gradual increase in the number of oxide particles and graphite nodules. In this mechanism, the oxides and graphite nucleation can occur at various stages during solidification, resulting in wide range of different sizes of oxides graphite nodules.

In a rapid cooling rate, there will be multiplication of oxide particles and their count will be high. At the early stages of solidification, there will be many nuclei present in the melt which can nucleate a large number of nodules at the same time and there will be a narrow size range in the graphite nodules. The latter case of fast cooling can be referred to the result in Figure 1, for sample I76-7, where there are tremendous large number of graphite nodules and there is very little size variation among the nodules. Due to rapid cooling, one can expect $\mathrm{MgO}$ multiplication during solidification which can nucleate graphite nodules at different steps. The slow cooling can be referred to sample I76-2 in the same figure. There are various sizes of nodules and much lower in number than I76-7. If the cooling rate is very slow then there will be a wide size range of nodules in the sample but due to the nearly equilibrium (thermodynamically) melt, $\mathrm{MgO}$ will not nucleate until the end of solidification. Thus oxides might nucleate homogeneously and graphite nodule will nucleate on them, hence resulting in colonies of different sizes of graphite nodules as shown in Figure 1 for 1150-3.

Case II: Variable cooling rate: Two type of mechanism can occur in this case; rapid cooling rate in the beginning for a short time but then drops quickly and rapid cooling till the eutectic temperature, then reduces abruptly. The first type of mechanism is explained by the result shown in Figure 1 for sample 1150-7. In the beginning, the cooling rate is faster due to the presence of four steel channels. This is shown in the simulated cooling curve for 1150-7 in Figure 2, in which the cooling rate reduces after $\sim 1250{ }^{\circ} \mathrm{C}$, and soon equilibrium conditions are met. Due to lower cooling rate, many of the small $\mathrm{MgO}$ particles will be unstable and dissolve back but few might survive as it approaches to the solidification point. Relatively few nodule will nucleate at the beginning due to less number of oxide particles available. They will continue to grow through the $\mathrm{C}$ diffusion but then the melt might start depleting with $\mathrm{C}$ and further nucleation of graphite ceases for a while until 
the conditions are met again. $\mathrm{MgO}$ will also continue to grow to nucleate homo-or heterogeneously during the solidification because of the enrichment, and there will be a local increase in the number of oxides particles. They will remain in the liquid until the graphite starts to precipitate again, and new nodules will start to nucleate on the existing oxide particles. This can happen either at the very end of solidification or slightly before that. That's why there are large segregation areas and different size of nodule colonies as explained in the schematic figure 5(a).

In the 2 nd type, the cooling rate is faster $(\sim 20 \mathrm{~K} / \mathrm{min},[5])$ in the beginning due to the steel channels nearby, but later when the heat flux reduces due to heat saturation at the steel channels, the cooling rate drops abruptly just before the eutectic temperature, as shown by the simulated cooling curve for 1150-4 in Figure 3. At the beginning of the eutectic reaction, many oxides will be present as nucleation sites resulting in large number of nodules. Then the liquid will become homogenized and will equilibrium conditions will be achieved due to very slow cooling. The remaining oxides (small ones) will get unstable and dissolve back into the melt. The existing graphite nodules will continue to grow through diffusion process. The carbon content in the melt might reduce to a low value due to a large population growth of nodules at the same time, graphite nucleation will be delayed until the last stage of solidification. However $\mathrm{MgO}$ will continue to nucleate homogeneously or heterogeneously at different solid fractions due to micro segregation. These oxides particles will remain in the liquid until graphite can be precipitated. As shown in Figure 1 (IP24- 4), there are many small size uniformly distributed nodules, but few intermediate nodules. This is explained in a schematic diagram in Figure 5(b).

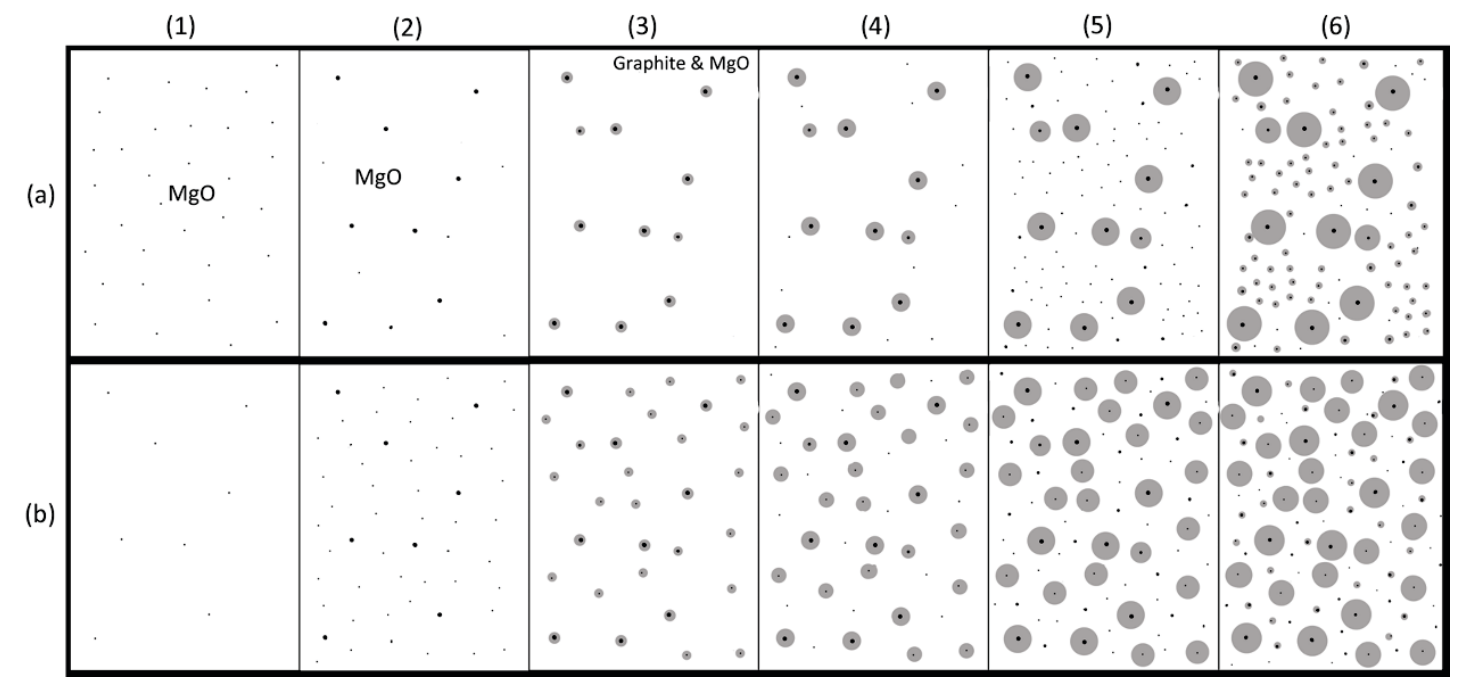

Fig. 5. Nucleation mechanism of $\mathrm{MgO}$ and graphite nodules. (a)-Fast cooling in the beginning but slow while approaching the freezing line. al- fast cooling, many $\mathrm{MgO}$, a2- slow cooling, most $\mathrm{MgO}$ dissolve, a3- solidification starts, graphite appears, a4- new $\mathrm{MgO}$ formed, a5- More $\mathrm{MgO}$ nucleated but low $\mathrm{C}$, a6- New homo-nucleation of nodules on $\mathrm{MgO}$. (b)- Fast cooling in the beginning till freezing point. $b 1-\mathrm{MgO}$ nucleated, $b 2$ - More $\mathrm{MgO}$ nucleated, $b 3$ - graphite nucleated and solidification started, $b 4-$ more $\mathrm{MgO}$ nucleated, $b 5-\mathrm{MgO}$ increased but no new nodules, b6- New $\mathrm{MgO}$ and new graphite nodules.

Similarly sample 1150-7 in Figure 2, there is a large difference between smallest and large size nodule. There are relatively many very small size nodules compared the ones in sample 3 (Figure 3). The reason for this is the same as mentioned above for sample 4; however the solidification is much slower than the position at sample 4. It is not shown in the cooling curve but the simulation have confirmed that this point is the last solidifying point which means, the segregation problem is more serious in this part of the casting. Calculation shows that by increasing the radius of the initial particle, the $\mathrm{Mg}$ level will reach nucleation line faster at even a slow cooling rate. In our future investigations, we could add external particles such as $\mathrm{SiO}_{2}$ to the melt during casting operation. Since $\mathrm{MgO}$ can immediately interact with the $\mathrm{SiO}_{2}$ particles and form either 
$\mathrm{MgSiO}_{2}$ or only $\mathrm{MgO}$ by releasing free $\mathrm{Si}$ or even a combination of both. This could enhance the nucleation process.

\section{Conclusion}

At higher cooling rates, oxides multiplication occurs due to multistep nucleation which affects the nodule count and homogenization. At a constant higher cooling rate, completely different nodule count and size distribution is obtained than in the variable cooling rate. A higher cooling rate prior to solidification till the starting point of solidification will results in numerous amount of $\mathrm{MgO}$ particles which can produce a single size large population of graphite nodules at the beginning of solidification. In large size casting, due to very slow cooling, micro segregation is dominant and controls the graphite morphology and size distribution.

\section{Appendix}

The interaction co-efficient of $\mathrm{O}$ and $\mathrm{Mg}$ on various elements in the liquid are given below.

Table 2. Interaction co-efficient of $\mathrm{O}$ and $\mathrm{Mg}$ on the important elements which can affect the results. Values in * are assumed in comparison to others. Whereas; $\varepsilon_{i}^{J}=A t_{w t}(i) \times e_{i}^{J} / 0.2425$ [7].

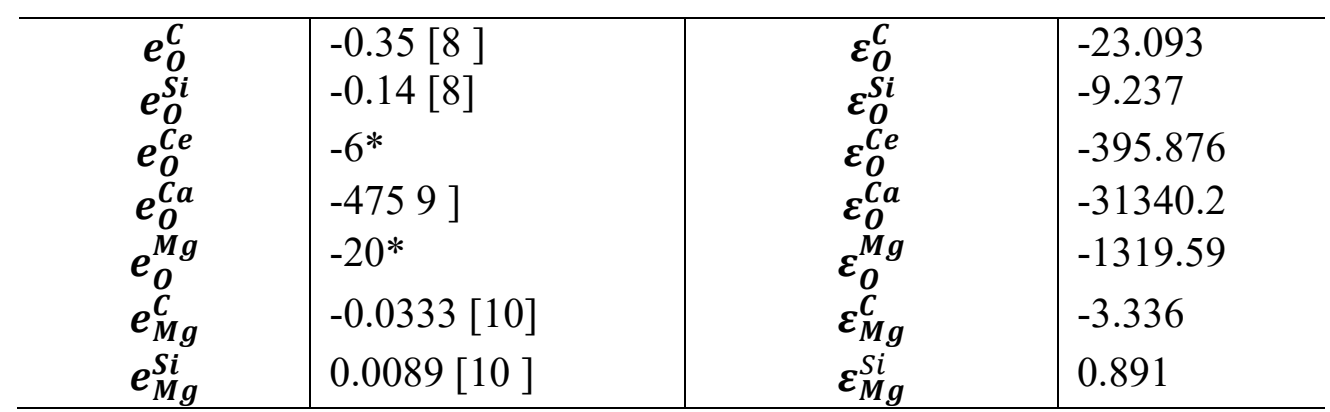

The interaction co-efficient and Gibbs free energy of $\mathrm{MgO}$ are calculated as below;

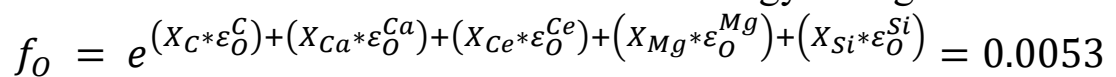

$$
\begin{aligned}
& f_{M g}=e^{\left(X_{C} * \varepsilon_{M g}^{C}\right)+\left(X_{S i} * \varepsilon_{M g}^{S i}\right)}=0.6504 \text {, } \\
& \Delta G=-4.1858(181600+7.37 \mathrm{~T} \log T-75.5 \mathrm{~T}) \mathrm{J} / \mathrm{mol}
\end{aligned}
$$

Since oxygen and $\mathrm{Mg}$ are equally consumed in the formation of $\mathrm{MgO}$ particles, therefore the equilibrium oxygen content along temperature was calculated analytically. The decrease in $\mathrm{Mg}$ along decreasing temperature will be also accompanied by the decrease of oxygen. The procedure to evaluate this decrease is to find a reduction factor by which oxygen will reduce step wise from an initial oxygen value. The equilibrium oxygen concentration will be

$$
\begin{aligned}
& X_{O}^{e q}=R f * X_{O}^{i-a t \text { inoc. } T}, \text { and } \\
& R f=\text { reduction factor }=\operatorname{sqrt}\left(X_{M g}^{e q} . X_{O}^{e q} / X_{O}^{i} \cdot X_{M g}^{e q}\right) .
\end{aligned}
$$

Whereas $X_{O}{ }^{i}$ is the value one step before the current temperature. At the inoculation temperature $X_{O}^{L}=X_{O}^{e q}=4.5 \times 10^{-7}$ at $1723 \mathrm{~K}$.

When a cost iron melt is $\mathrm{Mg}$ treated, the initial oxygen level will reduce to another level. This decease will be according to the equilibrium value of oxygen. From experimental results it was shown [11] that the oxygen content before $\mathrm{Mg}$ treatment was reduced from $3.05 \mathrm{e}-6$ to $4.5 \mathrm{e}-7$ in mole fraction. This decrease is a result of the $\mathrm{MgO}$ formation. The number of $\mathrm{MgO}$ particle that are formed by utilizing the oxygen that is equal to the initial ' $\mathrm{O}$ ' minus the equilibrium ' $\mathrm{O}$ ', is

$N_{i}=([M g w t . \%] * 219) / 4 \pi R^{3} * 3580 . \mathrm{R}$ is the radius of $\mathrm{MgO}$ that is assumed as $500 \mathrm{~nm}$ particle which is formed during $\mathrm{Mg}$ treatment. The radius $\mathrm{r}$ of the $\mathrm{MgO}$ particle was evaluated analytically by taking small steps in time. In the first place 'dr' was calculated for a constant time step ' $\mathrm{dt}$ '. Those values in equation 1, which are changing with temperature, are thus calculated with 
the time steps which can give us the growth rate at a certain cooling rate. This can be done in the following way:

$$
d r_{n}=D_{M g} *\left(V_{m}^{M g o} / V_{m}^{M g} r\right)\left(X_{M g}^{e q}-X_{M g(n-1)}^{L}\right) d t / 0.5
$$

At each temperature step, the radius will be; $R_{n}=R_{n-1}+d r_{n}$

Similarly at any temperature step or drop (T_step), the new $X_{M g(t)}^{L}$ will be following;

$$
X_{M g(t)}^{L}(1)=X_{M g}^{e q}\left(\left(T_{i}\right)-T_{\text {step }}\right)-\left(V_{M}^{M g} / V_{M}^{M g o}\right)\left(N(2 / 3) \pi R 1^{3} /\left(1-N(4 / 3) \pi R^{3}\right)\right)
$$

At the nucleation point where $X_{M g(t)}^{L}=X_{M g}^{L}$ (homo . nucleation)

$$
\begin{aligned}
& d r_{n 1}=D_{M g} *\left(V_{m}^{M g o} / V_{m}^{M g} r\right)\left(X_{M g}^{e q-a t T n 1}-X_{M g}^{L}(n 1-1)\right) d t / 0.5 \\
& 1^{\text {st }} \text { nucleation event: } X_{M g(t-1)}^{L}(n 1-1)=X_{M g}^{n e w-i n i t i a l-(1)}-\frac{V_{M}^{M g}}{V_{M}^{M g o}}\left(\frac{2 * N \frac{2}{3} \pi R_{n 1}^{3}}{1-2 * N \frac{4}{3} \pi R_{n 1}^{3}}\right)
\end{aligned}
$$

Whereas, $R_{n 1}=R_{n 1-1}+d r_{n 1}$. The initial value of $\operatorname{Mg}\left(X_{M g}^{n e w-i n i t i a l-(1)}\right)$ is chosen in such a way that fulfils the condition of $X_{M g(t)}^{L}(n 1-1)=X_{M g}^{e q-a t T n 1}$ where $\operatorname{Tn} 1$ is the temperature at which the first nucleation occurs. Similarly for the any nucleation event $(\mathrm{m})$;

$$
\begin{aligned}
& d r_{n m}=D_{M g} \frac{V_{m}^{M g o}}{V_{m}^{M g} r}\left(\frac{X_{M g}^{e q-a t T n m}-X_{M g}^{L}(n m-1)}{0.5}\right) * d t \\
& X_{M g(t)}^{L}(n m-1)=X_{M g}^{n e w-i n i t i a l-(m)}-\frac{V_{M}^{M g}}{V_{M}^{M g O}}\left(\frac{f * N \frac{2}{3} \pi R_{n m}^{3}}{1-f * N \frac{4}{3} \pi R_{n m}^{3}}\right)
\end{aligned}
$$

Whereas; $R_{n 2}=R_{n 2-1}+d r_{n 2}$. The value of ' $\mathrm{f}$ ' will be $2^{m}$, where ' $\mathrm{m}$ ' is mentioned above. The total number of $\mathrm{MgO}$ particles per $\mathrm{m}^{3}$ or per $\mathrm{mm}^{3}$ is,

$$
\begin{aligned}
& N=N i+\left(2 \times N i_{1 s t n u c}\right)+\left(4 \times N i_{2 n d n u c}\right)+\left(8 \times N i_{3 r d n u c}\right)+\left(16 \times N i_{4 s t n u c}\right)+ \\
& \left(32 \times N i_{5 \text { th nuc }}\right)
\end{aligned}
$$

Whereas $\mathrm{Ni}$ is the initial number of $\mathrm{MgO}$ particle that are formed during $\mathrm{Mg}$ treatment. At $0.05 \% \mathrm{Mg}$ addition, the number of $\mathrm{MgO}$ particles are $808 \mathrm{per} \mathrm{mm}^{3}$ or $8.1 \times 10^{11} \mathrm{per} \mathrm{m}^{3}$. To perform these calculation for a different cooling rate, another time step $\mathrm{dt}(\mathrm{sec})$ is chosen.

\section{References}

[1]. T. Kobayashi, Strength and Toughness of Materials, 171-172, 2004, Springer-Verlag Tokyo, Japan, ISBN 978-4-431-53973-5.

[2]. T. Skaland, Nucleation Mechanisms in Ductile Irons, Proc. AFS conf. 'Cast Iron Inoculation', Schaumburg, Illinois, 2005.

[3]. H. M. Muhmond \& H. Fredriksson: ( $\mathrm{PhD}$ thesis), On the Inoculation and Graphite Morphologies of Cast Iron, 2014, Royal Institute of Technology, Stockholm, ISBN 978-91-7595349-6.

[4]. H. M. Muhmond \& H. Fredriksson, Graphite Growth Morphologies in Cast Iron, Mat. Sci. Forum, 790-791 (2014), 607-614.

[5]. A. Tadesse: ( $\mathrm{PhD}$ thesis), On the Volume Changes during the Solidification of Cast Irons and Peritectic Steels, 2017, Royal Institute of Technology, Stockholm, ISBN 978-91-7729-299-9. [6]. I. L. Svensson and I. Dugic, Modelling of volumes in cast iron solidification to predict shrinkage and expansion defects, Int. J. Cast Met. Res., 11-6 (1999), 489-494.

[7]. G. K. Sigworth \& J. F. Elliott, The Thermodynamics of Liquid Dilute Iron Alloys, 8 (iss.1) (1974), 298-310, MIT.

[8] J. F. Elliott, M. Gleiser and V. Ramakrishna, Thermochemistry for Steelmaking, 2 (1963), 547567. 
[9]. A. Ghosh and G. V. R. Murthy, An Assessment of Thermodynamic Parameters for Deoxidation of Molten Iron by Cr, V, Al, Zr and Ti, Transaction ISIJ, 26 (1986), 629-637.

[10]. V. Vondrák, J. Hampl, A. Hanus, Metallurgy of Cast Irons Out-of-Furnace Processing of Molten Cast Iron, 2014, 92-93, Technical University of Ostrava, Czech Republic.

[11]. F. Mampaey, D. Habets, J. Plessers, F. Seutens, The use of oxygen activity measurement to determine optimal properties of ductile iron during production, International Journal of Metalcasting, 4(2) (2010), 25-43. 